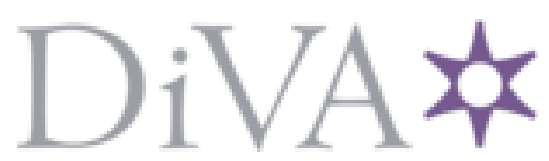

http://www.diva-portal.org

This is the published version of a paper published in Nordic Pulp \& Paper Research Journal.

Citation for the original published paper (version of record):

Hyll, C., Vomhoff, H., Nygårds, M. (2012)

Analysis of the plastic and elastic energy during the deformation and rupture of a paper sample using thermography.

Nordic Pulp \& Paper Research Journal, 27 (2): 329-334

http://dx.doi.org/10.3183/NPPRJ-2012-27-02-p329-334

Access to the published version may require subscription.

N.B. When citing this work, cite the original published paper.

Permanent link to this version:

http://urn.kb.se/resolve?urn=urn:nbn:se:kth:diva-104882 


\section{Analysis of the plastic and elastic energy during the deformation and rupture of a paper sample using thermography}

Caroline Hyll, Hannes Vomhoff, och Mikael Nygårds

KEYWORDS: Thermography, Paper mechanics, Emittance, Thermal energy, Rupture work, Plastic deformation, Elastic deformation.

SUMMARY: Thermography has been used to quantitatively analyze the plastic and elastic energy during deformation of paper. Sack paper samples were subjected to uniaxial tensile testing until rupture occurred. The temperature of the sample was simultaneously recorded with an infrared camera. The mechanical energy invested in the deformation was determined based on the force and deformation data. The thermal energy that accumulated in the sample during testing was estimated using the temperature measurements. Here, special attention was put on using the correct emittance values for the sack paper by measuring it with a new method. When comparing exerted mechanical energy with released thermal energy up to the time of sample rupture, about $40 \%$ to $60 \%$ of the mechanical energy could be detected as thermal energy. The lacking share of heat was most likely lost due to cooling of the sample during the experiments, as a lower share of detected mechanical energy was obtained for longer experiments. When comparing the increase in thermal energy during rupture to the elastic energy stored in the sample, an agreement of better than $90 \%$ was found.

ADDRESSES OF THE AUTHORS: Caroline Hyll (caroline.hyll@innventia.com): Innventia AB, Drottning Kristinas Väg 61, SE-114 28, Stockholm, KTH Royal Institute of Technology, Dept. of Production Engineering, Brinellvägen 68, SE-100 44 Stockholm, Sweden. Hannes Vomhoff (hannes.vomhoff@innventia.com) and Mikael Nygårds (mikael.nygards@innventia.com): Innventia AB, Drottning Kristinas Väg 61, SE-114 28, Stockholm. Corresponding author: Caroline Hyll

Thermography is a technique for non-destructive inspection, evaluation and testing of materials and processes. Within the pulp and paper industry, it has been used on many occasions, for example for analysis of the production process (Sheahen 1978; Vickery et al. 1978; Charles 1999), or investigations related to different product properties (Dumbleton et al. 1973; Kiiskinen and Pakarinen 1998; Hojjatie et al. 2001). Recent development in camera technology has made cameras with significantly higher spatial resolution and imaging speed available (Vollmer and Möllmann 2010).

In thermography, infrared detectors are used to measure the radiation emitted by objects due to their temperature (e.g. DeWitt and Nutter 1988, Vollmer and Möllmann 2010). The radiation can be converted to a temperature measurement using Planck's law. Here, the emitted radiation, the wavelength of the light and temperature are related for an ideal radiator, i.e. a blackbody. However, nearly all real objects are only emitting a fraction of the radiation that a blackbody would emit under identical conditions. This fraction, the emittance $\varepsilon$, is a parameter unique to each material. It has values between 0 (nonemitting) and 1 (blackbody). The emittance is a necessary input parameter to infrared cameras in order to obtain temperature values.

Using the correct value for the emittance is crucial, as an absolute error of $15 \%$ in the emittance could introduce an error of $3 \%$ regarding the absolute temperature (in Kelvin) of the object (Minkina and Dudzik 2009). For example, using an emittance of 0.82 when the true emittance is 0.71 would impart an error of $10 \mathrm{~K}$, if the measured object temperature is $343 \mathrm{~K}\left(70^{\circ} \mathrm{C}\right)$. An error in the assumed emittance value will have the largest impact on absolute temperature values; however it will also have a significant effect on measured temperature differences (Madding 2000).

Several methods may be used to measure emittance. However, there is no standardized method for measuring the emittance of paper. For a summary of different emittance measurement methods, see e.g. Madding (1999). Additionally, there are only a few experimental studies where the emittance of paper has been measured in detail. Hyll et al. (2011) developed a new method to measure the emittance of paper. Dependent on the type of paper and the investigated wavelength range, values in the range between 0.61 and 0.87 were obtained.

Thermography has also been used to analyze the deformation of a paper sample. In their pioneering work, Dumbleton et al. (1973) performed tensile testing of paper samples while filming the samples with an infrared line-scanner. The purpose was to investigate the temperature changes during a stress/strain experiment. They also compared the mechanical energy and thermal energy after failure. They found that 40 to $80 \%$ of the mechanical energy losses during tensile testing could be accounted for as increases in thermal energy. During the rupture of the sample, a large temperature increase was observed in the rupture zone, i.e. a large amount of energy was released. When comparing this energy with the stored elastic energy, some agreement was observed. The quantitative data spread however significantly. In this work, neither the emittance value is stated, nor is it mentioned in their section on possible measurement error sources. This allows the speculation that the authors were at that time not fully aware of the importance of the emittance value.

In a Master thesis work by Lidvall (1988) a 2D infrared camera was used to study the heat generated during loadreload tensile testing of paper. They noted that the zones 
where heat accumulated prior to the rupture did not always coincide with the rupture zone.

The research group of Yamauchi, Murakami and Tanaka performed several thermographic studies using a 2D infrared camera (Yamauchi and Murakami 1992; Yamauchi et al. 1993; Tanaka et al. 1997; Tanaka and Yamauchi 1997; Tanaka and Yamauchi 1999; Yamauchi and Hirano 2000; Tanaka and Yamauchi 2003; Tanaka and Yamauchi 2005). They studied mainly the temperature field in a paper sample during straining, tearing of paper samples and in the proximity of a notch. In one report on the straining of paper samples (Yamauchi and Murakami 1992), they show results, where the mechanical energy, expressed as TEA, is compared with an estimate of the thermal energy. In these particular results, these values agreed fairly well. Tanaka and Yamauchi (2003) quantitatively compared the tear energy and heat generation during the tearing of paper. They found that the ratio of heat generation of tear work was much smaller for tearing tests than for in-plane tensile tests; only around $10 \%$ of the tear work could be accounted for as thermal energy. The contribution of bending energy was suggested as one explanation. In only two of their publications, it is mentioned that the study was carried out using an infrared camera sensitive to wavelengths 8-13 $\mu \mathrm{m}$, with an assumed sample emittance of 1.0 (Yamauchi et al. 1993; Yamauchi and Murakami 1993).

In the previous studies, the approach was to determine the released mechanical energy based on the measured heating of the sample, i.e. the changes in temperature during the loading of the sample. As correct emittance values were not available in the previous studies, the quantitative results are likely to be erroneous. The objective of the present work was therefore to investigate if more quantitative information regarding the mechanical energy (in the form of temperature changes) can be obtained, if accurate emittance values are available. In particular, it was interesting to see if the plastic and elastic energy could be identified based on the thermographic measurements.

\section{Materials and Methods}

A uniaxial tensile test was performed on an industrially manufactured sack paper sample with a grammage of 68 $\mathrm{g} / \mathrm{m}^{2}$. The sack paper was assumed to have a specific heat $\mathrm{c}_{\mathrm{p}}=1.33 \mathrm{~kJ} / \mathrm{kgK}$, based on measurements on pine kraft paper by Kiiskinen et al. (1998). The tensile test was performed both in MD and $\mathrm{CD}$ direction. During the tensile test, the temperature of the samples was measured using an infrared camera. Using the force/deformation and temperature measurements, the mechanical and thermal energy, respectively, as a function of time were determined.

The emittance of the sample was determined using the "Directional Emittance Measurement" method, see Fig 1 and Fig 2 (Hyll et al. 2011). The "Directional Emittance Measurement" method is based on the reference emitter methodology, i.e. the radiation from a sample is compared to that from a reference with known emittance under otherwise identical conditions. Paper samples and

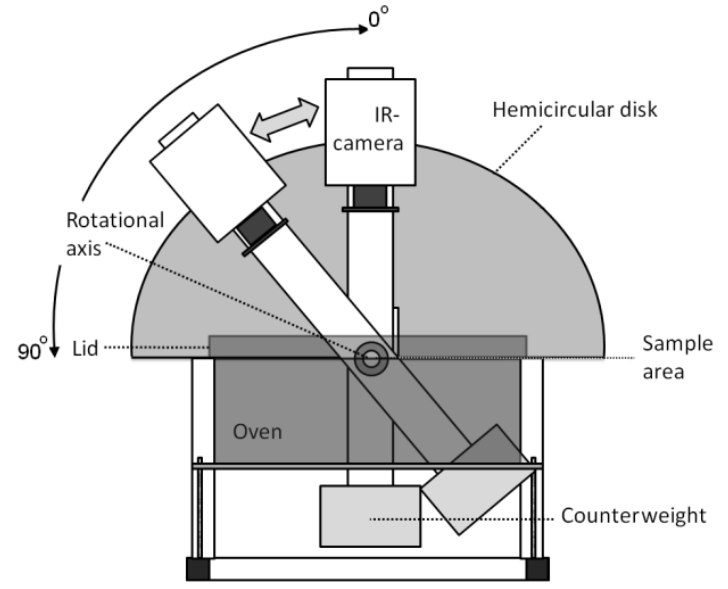

Fig 1. Illustration of "Directional Emittance Measurement" equipment.

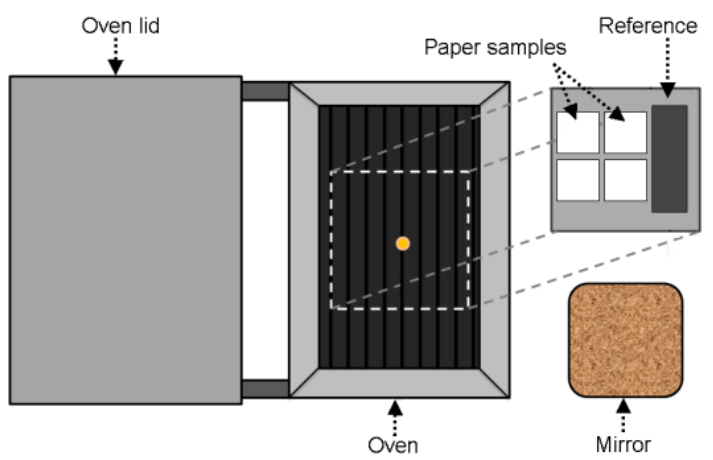

Fig 2. Illustration of oven Interior, samples, reference and infrared mirror.

emittance reference are placed inside an oven whose temperature can be controlled. The infrared camera is mounted onto a holder that can be pivoted around the oven in order to change the observation angle. When the desired oven temperature is reached, the lid of the oven is opened and the samples are imaged with the infrared camera.

Ambient radiation emitted from the surroundings is measured by imaging an infrared mirror at the same position as the samples. The emittance of the sample material can then be determined based on the measured radiation of the sample, mirror, and reference. In the present study the emittance was measured with the SC 6000 camera (sensitive at wavelengths 3.0-5.0 $\mu \mathrm{m}$, accuracy $\pm 2.0^{\circ} \mathrm{C}$ ), at normal incidence angle $\left(0^{\circ}\right)$. Hyll et al. (2011) found that the emittance of paper was independent of temperature, but that emittance measurements at a higher temperature had a higher accuracy. The emittance was therefore measured at a temperature of $70^{\circ} \mathrm{C}$. Further details regarding the DEM method are described in Hyll et al. (2011).

The uniaxial tensile test was performed with a Material Testing System (MTS). The MTS consisted of a servohydraulic cylinder whose movements were controlled. In the present configuration in the experiments, it was equipped with a load cell with a maximum load of 2500 $\mathrm{N}$, and a maximal cylinder displacement of $50 \mathrm{~mm}$. The 
study was performed in a climate controlled room with an air temperature of $23.0^{\circ} \mathrm{C}$ and $50 \%$ humidity.

The paper samples were cut in $50 \mathrm{~mm}$ wide and $140 \mathrm{~mm}$ long stripes and fastened in the MTS using clamps, see Fig 3. The distance between the clamps was $100 \mathrm{~mm}$ before the start of the experiments. When performing the tensile tests, several samples ruptured close to the clamps, so that the crack propagation could not occur freely. These tensile tests were not used for evaluation.

The infrared camera was mounted on a tripod, allowing an observation of the sample at a normal observation angle (distance approx. $0.5 \mathrm{~m}$ ). Cartonboard sheets were put around the equipment to shield it from infrared radiation sources in the room. However, not all external heat sources could be shielded away. In order to estimate the reflected ambient radiation from the surroundings, a golden mirror was put in front of the sample and imaged with the camera. The reflected ambient temperature was determined to be $24.7^{\circ} \mathrm{C}$.

The speed of the upper clamp during the experiments was set to $0.3 \mathrm{~mm} / \mathrm{s}$, i.e. the samples were deformed at a strain rate of $0.3 \% / \mathrm{s}$. The data sampling rate of the MTS was $102 \mathrm{~Hz}$. The infrared images were recorded with a frame rate of $32 \mathrm{~Hz}$ for the MD samples and $55 \mathrm{~Hz}$ for the CD samples.

During the measurement procedure, the MTS was started first, and the thermographic recording was activated shortly thereafter. Thus, dependent on experiment, the first 0.2 to 2.0 seconds of the deformation were not recorded with the infrared camera. The MTS was automatically stopped when reaching a set time (20 seconds for experiments in MD and 30 seconds for the experiments in CD). The camera recording was then stopped. A total of three MD samples and three CD samples were evaluated.

When evaluating the experiments, the mechanical energy, applied by the MTS, was compared with the generation of thermal energy, based on the temperature measurement of the sample.

When evaluating the temperature of the samples as a function of time, the sample area closest to the clamps was excluded due to local heating in the proximity of the clamps, see Fig 3. The evaluation area had a size of $50 \mathrm{x}$ $90 \mathrm{~mm}$. The average and maximum temperature of the samples were determined. When calculating the temperature values, the emittance, reflected ambient temperature, distance between object and sample, and air temperature were accounted for.

The MTS output was given as load $[\mathrm{N}]$ vs. displacement of the upper clamp [mm]. As the time scale of the camera recording and MTS data were not the same, the data were re-sampled using a Matlab routine to the same sampling rate. Given that the camera recording and the MTS displacement were not activated simultaneously, the data sets were aligned as a function of time under the assumption that the maximum temperature of the sample coincided with the time of failure, i.e. the maximum forces applied to the sample.

An example of a determined average temperature is depicted in Fig 4. From this measurement, the increase in
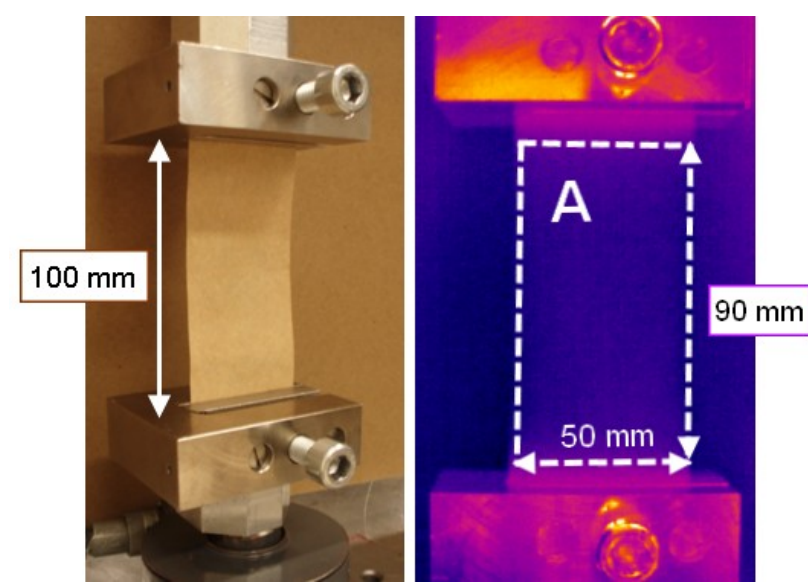

Fig 3. Visual image of sample clamped to the MTS (left) and infrared image of a sample (right) with illustration of the area " $\mathrm{A}$ " over which the average temperature was determined.

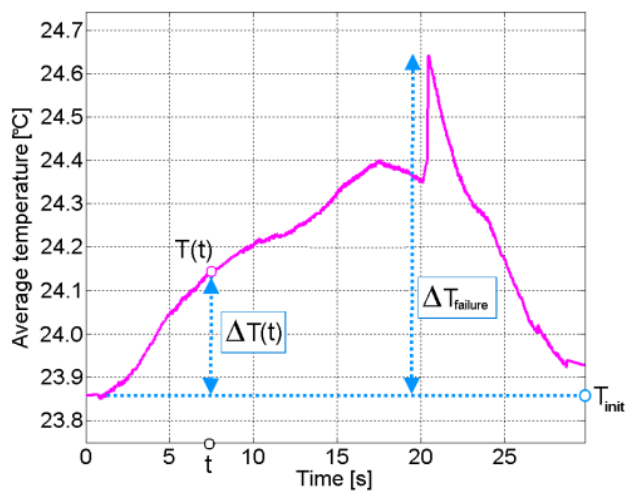

Fig 4. Example of a measured average temperature and the determined average temperature increase $\Delta T(t)$.

temperature as a function of time, $\Delta \mathrm{T}(\mathrm{t})$, was determined. The thermal energy $E(t)_{\text {therm }}$ that was accumulated in the sample during the deformation process was approximated at each time point $\mathrm{t}$ :

$$
\mathrm{E}(\mathrm{t})_{\text {therm }}=\mathrm{c}_{\mathrm{p}} \mathrm{Aw} \Delta \mathrm{T}(\mathrm{t}) \quad[\mathrm{J}]
$$

where $c_{P}[\mathrm{~kJ} / \mathrm{kgK}]$ is the specific heat, $A\left[\mathrm{~m}^{2}\right]$ is the evaluated area of the sample, $\mathrm{w}\left[\mathrm{g} / \mathrm{m}^{2}\right]$ is the grammage $\left[\mathrm{g} / \mathrm{m}^{2}\right]$, and $\Delta \mathrm{T}(\mathrm{t})[\mathrm{K}]$ is the increase in average sample surface temperature as a function of time. Possible losses of energy due to convection and radiation of heat, or the evaporation of water were not accounted for in the calculations but are partly addressed in the discussion section. Furthermore, an eventual change in grammage in the observed area as a result of the sample deformation was also not considered.

The total mechanical energy $E(t)_{\text {mech }}$ added to the sample during the deformation was estimated as

$$
\mathrm{E}(\mathrm{t})_{\text {mech }}=\int_{0}^{\mathrm{s}(\mathrm{t})} \mathrm{F}(\mathrm{t}) \mathrm{dS} \quad[\mathrm{J}]
$$

where $F(t)[N]$ is the applied force and $s(t)[\mathrm{m}]$ is the deformation of the sample. Furthermore, the plastic and elastic energies were approximated, see illustration in Fig 5.

The plastic energy (area A) was obtained by subtracting the elastic energy (area B) from the total mechanical energy. Here, it was assumed that the plastic energy also 


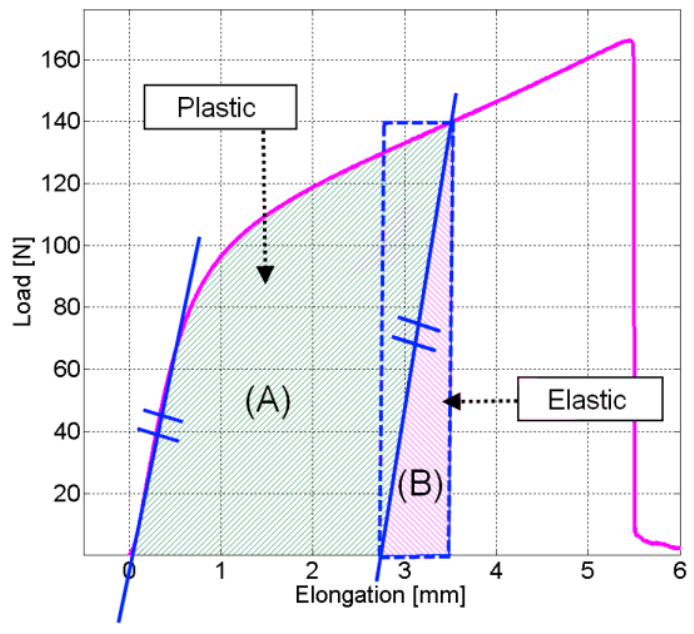

Fig 5. Plastic (area A) and elastic (area B) mechanical energy, illustrated at time $\mathrm{t}=3.5 \mathrm{~s}$.
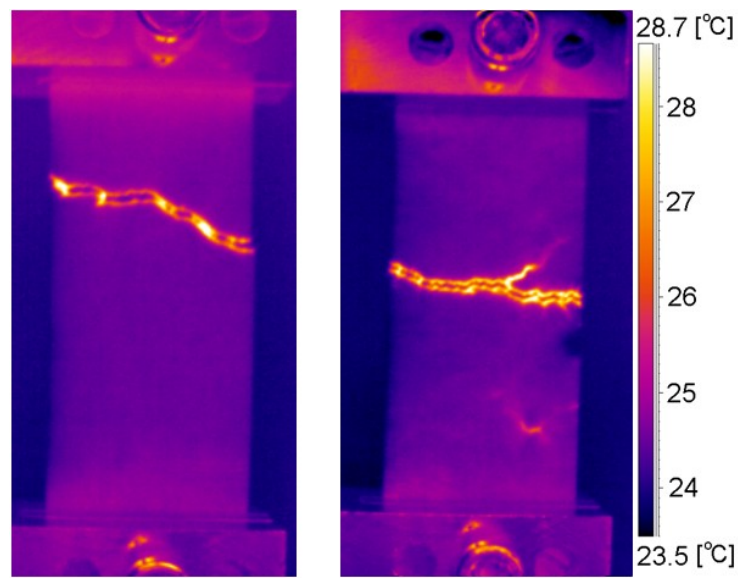

Fig 6. Infrared image of paper samples at time of failure. Left: MD sample. Right: CD sample.

included possible viscous effects. When calculating the triangle representing the elastic energy at a certain time $t$, the slope of the curve in the elastic regime in the beginning of the deformation curve was used.

\section{Results}

The emittance $\varepsilon$ of the sack paper samples was measured to be $0.76 \pm 0.03$. This value is considerably lower than 1.0 that was assumed by Yamauchi, Murakami and Tanaka in their studies with their bleached softwood kraft handsheets. The influence of emittance on the accuracy of the energy estimations will be discussed later in this section.

An example of typical force vs time and maximum temperature vs time plots are depicted in Fig 7. During deformation the average temperature increased gradually. When the sample reached failure a sharp increase in maximum temperature in the order of $6^{\circ} \mathrm{C}$ was observed. This increase in maximum temperature occurred in the rupture zone where a lot of energy was dissipated in a relatively short time, see thermographic images of two ruptured samples in Fig 6. The left image in Fig 6 (the MD sample) shows a well-defined deformation crack and an even temperature distribution in the rest of the sample. In contrast to that, the right image (CD sample) exhibits, apart from the higher temperature in the crack

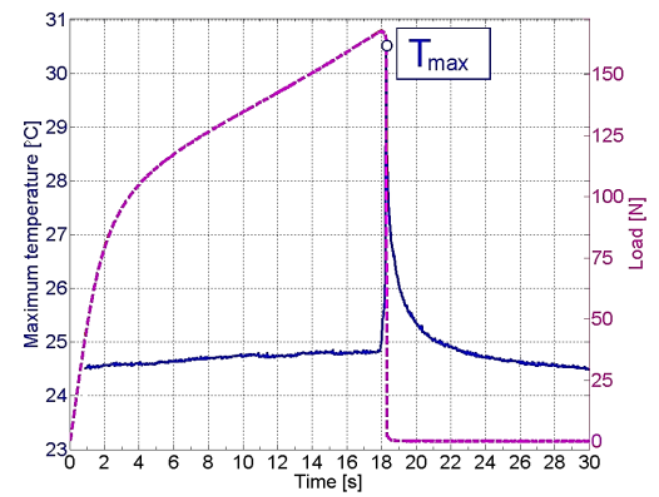

Fig 7. Maximum measured temperature of an MD sample (solid line) and load of MTS (dashed line).

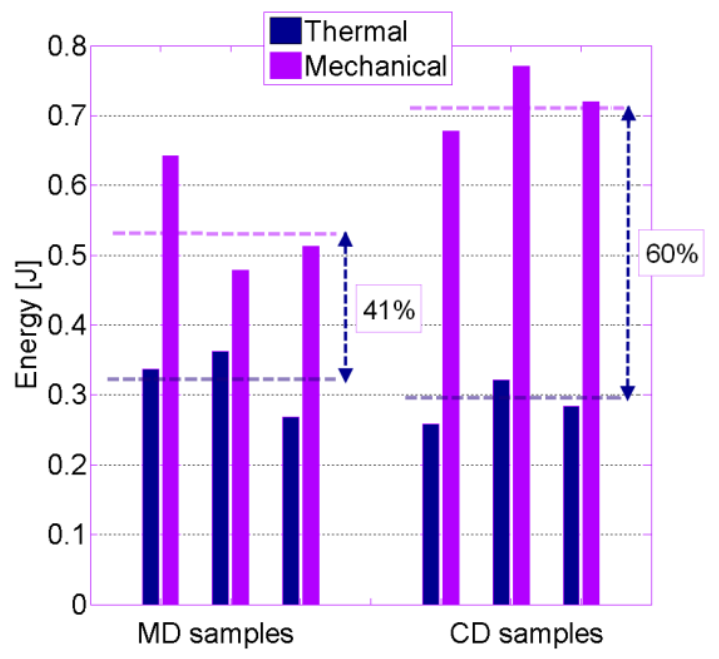

Fig 8. Thermal energy and mechanical energy at time of failure. Mean values indicated by dashed lines.

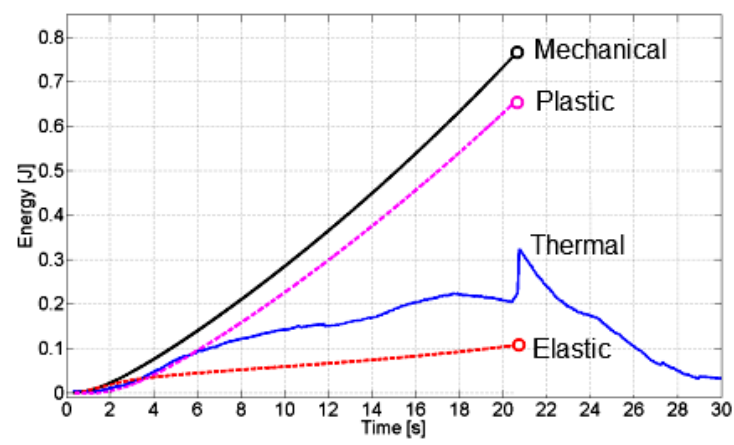

Fig 9. Thermal energy as a function of time, and mechanical, elastic and plastic energy until time of failure; CD sample.

zone, several local regions with significantly higher temperature at the time of failure.

The calculated thermal and mechanical energy during the entire deformation, are compared in Fig 8.

The calculated thermal energy averages differed from the mechanical energy averages by $41 \%$ and $60 \%$ for the MD and CD samples, respectively.

More information regarding the dissipation of energy can be obtained when looking at the thermal and mechanical energy as a function of time. Examples for a CD and a MD sample are shown in Fig 9 and Fig 10. 


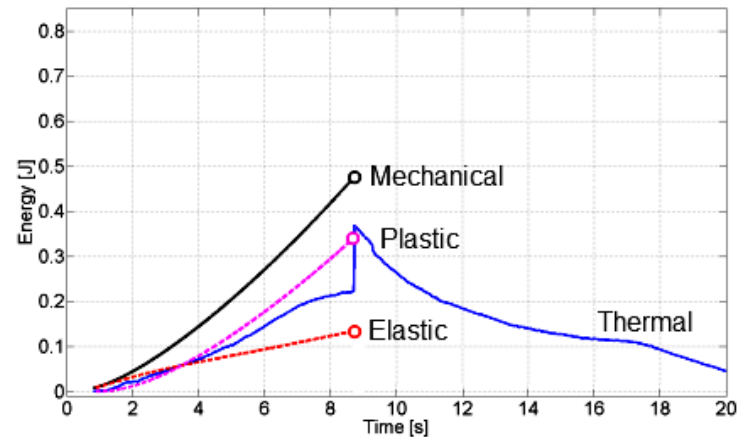

Fig 10. Thermal energy as a function of time, and mechanical, elastic and plastic energy until time of failure; MD sample.

In both cases, the mechanical energy increased steadily, with the plastic energy accounting for an increasing share. Initially the thermal energy followed the plastic energy. With increasing time, and consequently also with increasing average temperature, the difference between the plastic and the thermal energy became increasingly larger. The difference between thermal and plastic energy was larger for the CD-sample than the MD-sample.

During the rupture, a sudden increase in thermal energy was observed. Interestingly, for both samples, this increase was approximately equal to the estimated amount of elastic energy in the samples. This result corroborates the speculations by Ebeling (1970), Dumbleton et al. (1973), and Tanaka and Yamauchi (2005) that a large part of the elastic energy is expended during the rupture.

Just before the point of failure the temperature remained constant, or even showed a slight decrease. Here it seems that the rate of heat generation due to plastic deformation is lower than the heat loss due to radiation and convection, or possibility also the evaporation of moisture in the sample. As the experiment with the CDsamples lasted longer, more thermal energy was lost to the environment, which explains the larger difference for the CD samples, see Fig 8. This appears realistic, when one looks at the speed of cooling of the sample, i.e. the decrease in thermal energy, after the rupture of the sample. Here, a large amount of the accumulated energy is transferred to the environment within a matter of a few seconds. The rate at which the energy is transferred to the environment is initially in the order of $0.1 \mathrm{~J} / \mathrm{s}$ at the present temperature differences. This is twenty times more than the error estimation presented by Dumbleton et al. (1973). This illustrates, that the cooling of the sample during the deformation needs to be considered when evaluating the plastic energy.

As stated above, it was noted that the elastic energy at the time of failure was about the same as the sudden rise in thermal energy that resulted from the breakage. The results for the different experiments are compared in Fig 11. Apart from one MD sample, the elastic energy and the sudden increase in thermal energy deviated by less than $10 \%$.

Finally, we wanted to investigate the impact of the emittance on the results. Table 1 presents a comparison of the thermal energy at the time of failure, calculated using a) the measured emittance, and b) an assumed emittance of one.

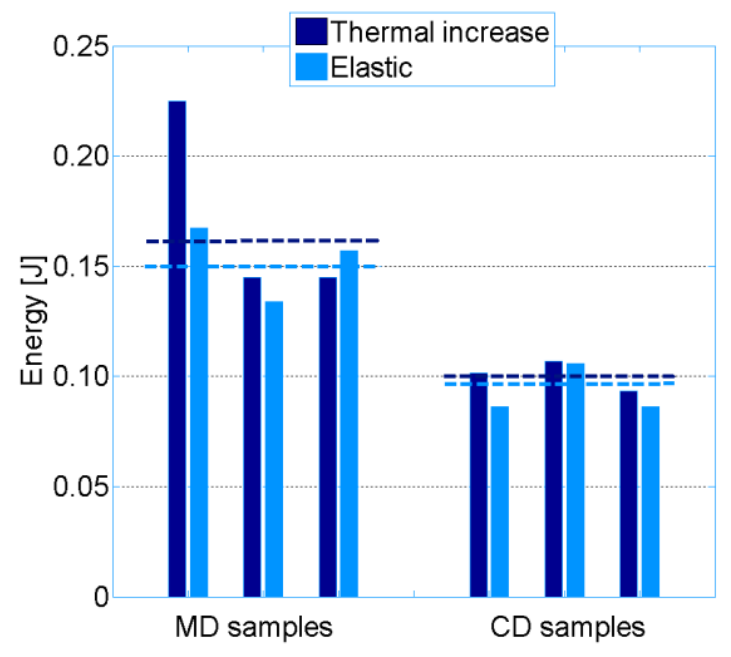

Fig 11. Increase in thermal energy compared to value of elastic energy at time of failure. Mean values indicated by dashed lines.

Table 1. Comparison of calculated thermal energy for emittance values of 0.76 and 1.00 .

\begin{tabular}{llll}
\hline Thermal energy [J] & $\boldsymbol{\varepsilon}=\mathbf{0 . 7 6}$ & $\boldsymbol{\varepsilon}=\mathbf{1 . 0 0}$ & Difference \\
\hline Avg. CD samples & $0.29 \pm 0.03$ & $0.22 \pm 0.03$ & $24 \%$ \\
Avg. MD samples & $0.32 \pm 0.05$ & $0.25 \pm 0.05$ & $22 \%$ \\
\hline
\end{tabular}

The calculated thermal energy differed by approximately $25 \%$, which illustrates the importance of using the correct emittance value when using the quantitative temperature values in thermography for the estimation of energies.

\section{Conclusions}

Infrared thermography has been used to study the energies that are dissipated during an uniaxial tensile test of industrial sack paper samples. In order to get a high accuracy for the measured temperature data, the emittance of the paper sample was determined using a new measurement method.

The thermal energy generated during the deformation was estimated from the thermographic data. The total mechanical, plastic, and elastic energy exerted during the deformation were calculated from the load-elongation curve.

From the start of the tensile test and up to the rupture of the sample, 40 to $60 \%$ of the exerted mechanical energy could be accounted for as released thermal energy, measured through an increase in the sample temperature. The remaining energy was most likely transferred already during the experiment to the environment due to cooling of the sample.

During the rupture of the samples, a sharp temperature increase occurred. Interestingly, the corresponding thermal energy increase was observed to correspond well to the elastic energy that was estimated to be stored in the sample at the time of rupture.

In the future, several methodological improvements could be made. Thermographic recording should be activated at the same time as the tensile testing. Also, finding a methodology to compensate for the cooling of the specimen during the tensile test would be useful, in 
order to measure a larger share of the plastic energy. Here, the cooling rate of the sample after the experiments could be of help in order to estimate the energy transfer to the environment for given experimental conditions. Furthermore, the temperature field measurements could be analyzed with a focus on their non-uniformity, especially when analyzing machine-made paper. Here, as indicated in previous publications, the plastic energy is generated non-uniformly as a result of the structural nonuniformity of the sample. By using correct values of the emissivity, quantitative information regarding the nonuniformity could be obtained. Finally, it would also be interesting to use the thermographic methodology to investigate how the plastic energy is dissipated in a twoply paper product, for example a white topliner that has different mechanical properties in the two plies. In such an experiment, a two-mirror arrangement could be used to monitor simultaneously the temperature changes on the front and back side.

\section{Acknowledgements}

The authors of this work would like to thank Anton Hagman and Sune Karlsson for their help with the experimental work. The comments by Johan Alfthan regarding the manuscript are most gratefully acknowledged.

\section{Literature}

Charles, J. A. (1999): Line-scan infrared imaging for papermachine diagnostics, 1999 TAPPI Engineering Conference, Anaheim, California, 10.

Dewitt, D. P. and Nutter, G. D. (1988): Theory and Practice of Radiation Thermometry, Wiley-Interscience, New York, p 1138. Dumbleton, D., Kringstad, K. and Söremark, C. (1973): Temperature profiles in paper during straining, Svensk Papperstid. (14), 521-528.

Ebeling, K. I. (1970): Distribution of Energy Consumption During Straining of Paper, Ph.D. Thesis, The Institute of Paper Chemistry, Appleton, Wisconsin, p 680.

Hojjatie, B., Abedi, J. and Coffin, D. W. (2001): Quantitative determination of in-plane moisture distribution in paper by infrared thermography, Tappi J. 84 (5), 11.

Hyll, C., Vomhoff, H. and Mattsson, L. (2011): A method for measurement of the directional emissivity of paper; Innventia Report 286, Innventia AB, Stockholm, p 40.

Kiiskinen, H., Lyytikainen, A. and Hamalainen, P. (1998): Specific heats of dry Scandinavian wood pulps, J. Pulp Pap. Sci. 24 (7), 5.

Kiiskinen, H. and Pakarinen, P. (1998): Infrared thermography at examination of paper structure, Proceedings of SPIE 3361, 6. Lidvall, F. (1988): Förändringar av luftresistans med hjälp av initierade deformationer av papper, Master Thesis, KTH Royal Institute of Technology, Stockholm, p 31.

Madding, R. P. (1999): Emissivity measurement and temperature correction accuracy and considerations, SPIE (ed.), SPIE Conference on Thermosense XXI, Orlando, Florida, 393-400.

Madding, R. P. (2000): Common misconceptions in infrared thermography condition based maintenance applications, Orlove, G. (ed.), InfraMation 2000, Orlando, FL, 229.

Minkina, W. and Dudzik, S. (2009): Infrared Thermography Errors and Uncertainties, John Wiley \& Sons Ltd, West Sussex, UK, p 190.
Sheahen, T. P. (1978): Use of infra-red thermography in pulp and paper mills, TAPPI ANNUAL MEETING (Chicago), 1.

Tanaka, A., Otsuka, Y. and Yamauchi, T. (1997): In-plane fracture toughness testing of paper using thermography, Tappi $80(5), 5$

Tanaka, A. and Yamauchi, T. (1997): Size Estimation Of Plastic Deformation Zone at the Crack Tip of Paper under Fracture Toughness Testing, J. Pack. Sc. Technol. 6 (5), 8.

Tanaka, A. and Yamauchi, T. (1999): Examination of the essential work of fracture method for paper, 1999 International Paper Physics Conference, Atlanta, GA, TAPPI Press, 171-177

Tanaka, A. and Yamauchi, T. (2003): Heat generation under paper tearing, International Paper Physics Conference, Victoria, BC, Canada, Pulp and Paper Technical Association of Canada, 287-292

Tanaka, A. and Yamauchi, T. (2005): Thermographic observations of the out-of-plane tearing process of paper, Appita Journal 58 (3), 5.

Vickery, D. E., Luce, J. E. and Atkins, J. W. (1978): Infrared thermography - An Aid to solving paper machine moisture profile problems, Tappi Papermakers Conference, 39-51.

Vollmer, M. and Möllmann, K.-P. (2010): Infrared Thermal Imaging: Fundamentals, Research and Applications, WileyVCH, Weinheim, Germany, p 593.

Yamauchi, T. and Hirano, H. (2000): Examination of the onset of stable crack growth under fracture toughness testing of paper, Journal of Wood Science 46 (1), 5.

Yamauchi, T. and Murakami, K. (1992): Observation of deforming and fracturing processes of paper by using infrared thermography, Japan Tappi 4, 70-75.

Yamauchi, T. and Murakami, K. (1993): Observation of deforming and fracturing processes of paper by use of thermography, Products of Papermaking - Tenth Fundamental Research Symposium Oxford, UK, Leatherhead, UK: Pira International, 825-847

Yamauchi, T., Okumura, S. and Noguchi, M. (1993): Application of thermography to the deforming process of paper materials, Journal of Materials Science 28, 4549-4552. 